Titre :

Mésothéliome : les dispositifs en place en France "le réseau mésothéliome"

1998-2013

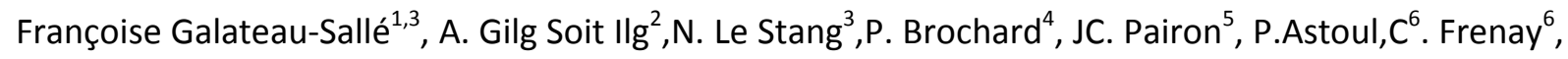
G Blaizot $^{1}$, S. Chamming's ${ }^{5}$, S. Ducamp ${ }^{2}$, T. Rousvoal ${ }^{1}$, A. de Quillacq ${ }^{1}$, V. Abonnet ${ }^{1}$, I Abdalsamad ${ }^{1}, H$ Begueret $^{1}$, E Brambilla ${ }^{1}$, FCapron ${ }^{1}$, MC Copin ${ }^{1}$, C Dane $^{1}$ I, AY de Lajartre ${ }^{1}$, A. Foulet-Roge ${ }^{1}$, L Garbe ${ }^{1}$, O Groussard $^{1}$, S Giusiano ${ }^{1}$, V Hofman ${ }^{1}$, S. Lantuejou ${ }^{1}$ I, JM Piquenot ${ }^{1}$, I Rouquette ${ }^{1}$, C Sagan ${ }^{1}$, F ThivoletBejui $^{1}$, JM Vignaud ${ }^{1}, A$. Scherpereel, ${ }^{7} M C$. Jaurand ${ }^{8}$, D. Jean ${ }^{8}$, P Hainaut ${ }^{9}$, L .Chérié -Challine ${ }^{2}, M$. Goldberg $^{2}$, D. Luce ${ }^{2}$,E. Imbernon ${ }^{2}$.

1. Centre National Référent cancers rares MESOPATH "mésothéliomes malins pleuraux et tumeurs péritonéales rares" CHU Caen Côte de Nacre, 14033 Caen cedex 9

2. Département Santé Travail, Institut de Veille Sanitaire, 12 rue du Val d'Osne, 94415 SaintMaurice Cedex France

3. Registre MESONATCHU CAEN Côte de Nacre, 14033 Caen cedex 9

4. ISPED-LSTE-ESSAT 146 Rue Leo Saignat, 33076 Bordeaux Cedex

5. I.I.M.T.P.I.F. CHI Créteil 40, avenue de Verdun -94010 CRETEIL Cedex et INSERM, U955, Créteil, France (JCP, PA); Université Paris-Est Créteil, France

6. Oncologie Thoracique, Maladies de la Plèvre et Pneumologie Interventionnelle, Hôpital Nord,Aix-Marseille Université, Marseille

7. Service de pneumologie et oncologie thoracique, hôpital Calmette, CHRU de LilleAvenue Oscar Lambret, 59037 Lille Cedex. Faculté de médecine Henri-Warembourg, université de Lille-II, unité Inserm 774, Lille.

8. INSERM, UMR-674, IUH, Paris, 75010, France

9. InternationalPreventionResearchInstitute, Lyon,France

Résumé:

Résumé : le réseau des mésothéliomes

Les mésothéliomes sont des cancers rares représentant moins de 0,3\% des cancers en France. II s'agit de cancers très agressifs, résistants à de nombreux traitements conventionnels et à forte implication médicosociale. Ce cancer lié à une exposition à l'amiante, est présente chez l'homme dans $80 \%$ des cas. À la fin des années 1990 , les projections réalisées à partir de la modélisation de la mortalité montraient que l'augmentation de l'incidence du mésothéliome pleural allait se poursuivre pendant deux à trois décennies. Le diagnostic des mésothéliomes est anatomopathologique, mais la présentation histologique revêt des aspects très variés et trompeurs, sources de difficultés diagnostiques. C'est devant un tel contextediagnostique, clinique, épidémiologique, et médicosocial, qu'un réseau national et international, multidisciplinaire sur le mésothéliome a été progressivement construit en France pour répondre soit à des questions de surveillance épidémiologique et/ou de recherche translationnelle ou plus académique, soit participer à la structuration de l'offre de soins en réponse à l'action 23.1 du Plan cancer des 2009 et ainsi assurer une meilleure prise en charge à 
visée sanitaire de cette pathologie. La structure de ce réseau multidisciplinaire et les résultats portant sur les 15 premières années d'existence, apportent la preuve de son intérêt et de son efficacité, pour produire des indicateurs de surveillance épidémiologique. Les résultats du réseau des pathologistes confirment l'intérêt de la relecture selon une procédure anatomopathologique de certification standardisée pour assurer la qualité des données épidémiologiques, la qualité de la reconnaissance médicosociale et la fiabilité du diagnostic clinique pour le bénéfice des patients.

Mots clé: mésothéliome, seconde lecture, impact diagnostique, impact médicosocial, impact thérapeutique, base clinicobiologique, registre de cancers.

Mesothelioma is a rare disease less than $0,3 \%$ of cancers in France, very aggressive and resistant to the majority of conventional therapies. Asbestos exposure is nearly the only recognized cause of mesothelioma in men observed in $80 \%$ of case. In 1990 , the projections based on mortality predicted a raise of incidence in mesothelioma for the next three decades. Nowadays, the diagnosis of this cancer is based on pathology, but the histological presentation frequently heterogeneous, is responsible for numerous pitfalls and major problems of early detection toward effective therapy. Facing such a diagnostic, epidemiological and medico legal context a national and international multidisciplinary network has been progressively set up in order to answer to epidemiological survey, translational or academic research questions. Moreover, in response to the action of the French Cancer Program (action 23.1) a network of pathologists was organized for expert pathological second opinion using a standardized procedure of certification for mesothelioma diagnosis. We describe the network organization and show the results during this last 15 years period of time from 1998-2013. These results show the major impact on patient's management, and confirm the interest of this second opinion to provide accuracy of epidemiological data, quality of medico legal acknowledgement and accuracy of clinical diagnostic for the benefit of patients. We also show the impact of these collaborative efforts for creating a high quality clinicobiological, epidemiological and therapeutic data collection for improvement of the knowledge of this dramatic disease.

Key words: mesothelioma, expert pathological second opinion, diagnostic impact, medicolegal impact, therapeutic impact, clinicobiological database, register of cancers.

\section{Date de création du réseau}

Date de création : 1998

\section{Description et but du réseau}

Le mésothéliome est une tumeur raredes séreuses, représentant 0,3\% de l'ensemble des cancers, ce qui situe cette pathologie au dernier rang des cancers dont l'incidence et la mortalité par cancer en France de 1978 à 2000 ont été étudiéspar le réseau Francim. II s'agit d'un cancer capable de revêtir de multiples aspects histologiques trompeurs, source de difficultés diagnostiques fréquentes. La pratique d'une double lecture pour cette pathologie est ancienne, puisque dès 1972 un collège spécialisé de pathologistes a été créé. A cette époque, 30\% des cas soumis à cette expertise collégiale étaient exclus de ce diagnostic. Ce cancer est de plusagressif, résistant à la majorité des traitements conventionnels même si de récentes avancées ont permis un gain de survie. Enfin lerôle de l'exposition à l'amiante, qu'elle soit d'origine professionnelle, paraprofessionnelle, domestique ou 
au cours d'activités de bricolage est connu: I'amiante est considéré comme le facteur étiologique responsable dans plus de $80 \%$ des cas de mésothéliomes pleuraux survenant chez l'homme dans les pays industrialisés. Chez la femme pour cette même localisation, le risque attribuable à l'exposition à l'amiante est inférieur à 50\%,sans qu'un autre facteur étiologique soit identifié. Les professions les plus exposées ont évolué avec le temps. Dans les années 60, les professions exposées concernaient les travailleurs des industries de l'extraction, de la transformation et de l'utilisation de l'amiante (1). Depuis les années 80, c'est parmi les travailleurs du bâtiment et de la maintenance que l'incidence de ce cancer est la plus élevée. À la fin des années 1990, les projections réalisées à partir de la modélisation de la mortalité montraient que l'augmentation de l'incidence du mésothéliome pleural allait se poursuivre pendant deux à trois décennies (2). Cependant, grâce aux mesures prises par certains pays, et en particulier par la France on peut voir l'absence d'augmentation attendue de l'incidence chez l'homme. Par contre, chez la femme on observe une augmentation de l'incidence des mésothéliomes pleurauxet on note que le taux annuel moyen d'évolution de l'incidence pour les 20 dernières années est le plus élevé de tous les cancers chez la femme $(+6,83 \%)(3,4)$.

Devant un tel contexte diagnostique, clinique, épidémiologique, et médicosocial, un réseau national et international, multidisciplinaire sur le mésothéliome a été progressivement construit en France pour répondre soit à des questions de surveillance épidémiologique et/ou de recherche translationnelle ou plus académique, soit participer à la structuration de l'offre de soins en réponseà l'action 23.1 du Plan cancer de 2009 et ainsi assurer une meilleure prise en charge à visée sanitaire de cette pathologie.

Ainsi, les travaux et l'effort de concertation développé dès 1998 par leProgramme National de Surveillance des Mésothéliomes-PNSM (Figure 1),par le registre multicentrique à vocation nationaledes mésothéliomes pleuraux -MESONAT (Figure 2), puis le centre national référent cancers rares : "mésothéliomes malins pleuraux et tumeurs péritonéales rares" -MESOPATH (Figures 3, 4a et 4b) et le Centre d'excellence international IM@EC (Figure 5) ont permis de constituer un pôle de recherche et de constituer la base clinico-biologique MESOBANK pour la recherche translationnelle et épidémiologique dans ce domaine (Figure 6), à fort potentiel international tant par le nombre de cas recensés ( $>16000$ dossiers patients depuis 1972) que par l'impact de ces activités sur les efforts internationaux de détection et de classification des mésothéliomes. Depuis janvier 2012, ce réseau a été complété par la mise en place de la déclaration obligatoire ( DO) des mésothéliomes.

\section{Fonctionnement et structures constituant le réseau}

\section{1-Le Programme National de Surveillance du Mésothéliome (PNSM)}

Le Programme national de surveillance du mésothéliome a été mis en place en 1998, à la demande des Ministères de la Santé (DGS) et du Travail (DGT), coordonné par le Département Santé et Travail (DST) de I'Institut de Veille Sanitaire (InVS). Ce Programme constitue un système de surveillance épidémiologique des effets de l'amiante sur la santé de la population française à travers le suivi permanent du mésothéliome pleural. L'organisation du PNSM repose sur plusieurs volets : 1 ) le volet Incidence ; 2) le volet Expositions - Etiologie ; 3) le volet Confirmation Anatomopathologique et Clinique ; 4) le volet Évaluation de la prise en chargeau titre des maladies professionnelles. Ce Programme de surveillance épidémiologique, concerne le suivi permanent du mésothéliome pleural. Chaque volet est coordonné par un Centre national. L'ensemble des procédures du PNSM a reçu l'agrément de la Commission Nationale de l'Informatique et des Libertés. 


\section{Les principaux objectifs du PNSM sont :}

- d'estimer l'incidence nationale du mésothéliome en France et son évolution ;

- d'étudier la proportion des mésothéliomes attribuables à une exposition à l'amiante, notamment d'origine professionnelle sur le territoire français ;

- De décrire des secteurs d'activité et des professions à risque pour la survenue du mésothéliome chez l'homme; les secteurs d'activité économique et les professions sont classés selon l'importance du risque de mésothéliome pleural (5).

- D'établir les fractions attribuables : la fraction de risque attribuable à une exposition professionnelle à l'amiante chez l'homme a été estimée (estimation sur les hommes enquêtés entre 1998 et $2006(5,6)$ globalement à $91 \%$ et chez la femme à $41 \%$; des analyses plus détaillées sont en cours.

- de contribuer à la recherche d'autres facteurs étiologiques éventuels (fibres céramiques réfractaires, laines minérales, radiations ionisantes, virus SV40...)

- de contribuer à améliorer le diagnostic anatomopathologique du mésothéliome (7);

- d'évaluer la reconnaissance du mésothéliome de la plèvre comme maladie professionnelle ; la mise en place effective du Fonds d'indemnisation des victimes de l'amiante a élargi le champ de cet objectif(8).

Le PNSM repose sur l'enregistrement exhaustif, depuis le 1er janvier 1998, des tumeurs primitives de la plèvre incidentes dans un nombre restreint de départements. En effet les caractéristiques socioprofessionnelles et démographiques de la population couverte par le PNSMsont proches de celles de la France entière (Figure 1).

Dans chacun des départements, une procédure active de signalement des cas de tumeur primitive de la plèvre est mise en place auprès de l'ensemble des structures médicales spécialisées, afin de garantir une connaissance exhaustive des cas dès que le diagnostic est porté. Une procédure standardisée de confirmation anatomopathologique et clinique du diagnostic est ensuite utilisée pour tous les cas signalés selon la procédure précédemment publiéepar M. Goldberg (9). Les notifications concernent donc un enregistrement exhaustif, continu de données standardisées, validées et sécurisées.

\section{Résultats}

Le tableau 1 correspond à l'activité du volet anatomopathologique des cas certifiés pour les départements du PNSM pour la période 1998-2013. Résultats enregistrés dans la base de données du PNSM pour les prélèvements pleuraux (7).

Tableau 1 : activité du volet anatomopathologique des cas certifiés du PNSM pour la période 19982012

\begin{tabular}{|c|c|c|c|c|}
\hline $\mathrm{Nb}$ de prélèvements par année de prélèvement & $1998-2002$ & 2003-2007 & 2008-2012 & Total \\
\hline$\overline{\text { Reçus }}$ & 1051 & 1368 & 1762 & 4181 \\
\hline Conclus & 1040 & 1348 & 1577 & 3965 \\
\hline Mésothéliomes malins & $74 \%$ & $82 \%$ & $87 \%$ & $82 \%$ \\
\hline Incertains & $9 \%$ & $8 \%$ & $7 \%$ & $8 \%$ \\
\hline Exclus pour autre diagnostic & $9 \%$ & $6 \%$ & $3 \%$ & $5 \%$ \\
\hline Exclus pour matériel insuffisant & $8 \%$ & $4 \%$ & $3 \%$ & $5 \%$ \\
\hline
\end{tabular}


160 La déclaration obligatoire des nouveaux cas de mésothéliome mise en place à la demande du 161 ministère chargé de la santé est obligatoire depuis le 16 janvier 2012(décret $n^{\circ}$ 2012-47 du 16 janvier

162

163

164

165

166

167

168

169

170

171

172

173

174

175

176

177

178

179

180

181

182

183

184

185

186

187

188

189

190

191

192

193

194

195

196

197

198

199

2012 JORF n0015 du 18 janvier 2012, page 994, texte $n^{\circ}$ 25)en France métropolitaine et dans les territoires ultramarins. Elle concerne tout médecin clinicien et pathologiste qui pose ce diagnostic, quel que soit le site anatomique. C'est la 31e maladie à déclaration obligatoire (MDO) en France. Les formulaires de notification, à adresser par le médecin déclarant à l'ARS selon la région d'exercice, sont téléchargeables sur le site de l'InVS (http://www.invs.sante.fr), dans le dossier thématique « DO des mésothéliomes ". Ce dispositif, également mis en place par le département santé travail de I'InVS vient compléter le PNSM, et ne se substitue pas au système de déclaration des maladies professionnelles.

Les objectifs de cette DO conformes à l'avis du Haut Conseil de la santé publique (HCSP) d'octobre 2010 sont de contribuer à la lutte contre ce cancer et :

- de renforcer la surveillance épidémiologique des mésothéliomes, notamment dans les zones non couvertes par le PNSM (70\% de la population) et pour les localisations hors plèvre (12 à $15 \%$ des mésothéliomes)

- $d$ ' améliorer la connaissance des cas liés à des expositions extraprofessionnelles et en particulier environnementales par la "réalisation, d'enquêteseffectuées par la Cellule inter-régionale d'épidémiologie de I'InVS ou par l'équipe PNSM locale dans les régions concernées. Ces enquêtes concernent 3 populations ciblées: les mésothéliomes hors plèvre, les femmes et les hommes de moins de 50 ans.

- d'améliorer les demandes de reconnaissance en maladie professionnelle ou auprès du FIVA (

Lors de la phase pilote d'installation de cette DO les pathologistes ont joué un rôle important puisque $74 \%$ des mésothéliomes étaient déclarés par les pathologistes et $39 \%$ par les pneumologues, chirurgiens et généralistes.

\section{3-Le registre multicentrique à vocation nationale sur le mésothéliome pleural MESONAT}

Le registre MESONAT a été mis en place en 2006, et requalifié en 2012. Ce registre MESONAT, représente, par sa couverture populationnelle et sa qualité d'enregistrement, un des plus importants systèmes internationaux d'enregistrement des mésothéliomes et a la vocation d'un enregistrement pérenne exhaustif de cas parfaitement validés sur le plan anatomopathologique.Sa couverture populationnelle est d'un peu plus de19 millions d'habitants en 2012 (soit 30\% de la population française).Les cas enregistrés sont des patients résidant dans les départements décrits ci-dessus qui ont présenté une première suspicion de mésothéliome pleural de 1998 à l'année en cours. Les départements participants sont au nombre de 22 (Figure 2):

Depuis 1998: Calvados (14), Dordogne (24), Doubs (25), Gironde (33), Isère (38), Landes (40), LoireAtlantique (44), Lot-et-Garonne (47), Manche (50), Orne (61), Pyrénées-Atlantiques (64), Bas-Rhin (67), Haut-Rhin (68), Somme (80).

Depuis 1999 : Bouches-du-Rhône (13), Var (83) et Seine-Saint-Denis (93) 
Depuis 2006 : Alpes-Maritimes (06), Haute-Corse (2B), Corse-du-Sud (2A) et Seine-Maritime (76)

Depuis 2011 : Lille et sa région (59).

Les notifications concernent donc un enregistrement continu de données standardisées, validées et sécurisées. L'ensemble des procédures du Registre MESONAT a reçu l'agrément de la Commission Nationale de l'Informatique et des Libertés.

Les objectifs du registre MESONAT sont multiples:

1-Dans le domaine de la santé publique (surveillance et évaluation)

Le registre représente un élément central du PNSM, et constitue l'outil de base du dispositif national de surveillance épidémiologique du mésothéliome à des fins de santé publique. Cette surveillance comporte notamment les volets :

- Estimation de l'incidence nationale : un ratio incidence/mortalité est établi à partir des données des départements couverts par le registre et appliqué aux données nationalesde mortalité par classe d'âge, France entière, chez les hommes et les femmes séparément.

- Amélioration du diagnostic anatomopathologique du mésothéliome.

Dans chacun de ces domaines, les analyses sont répétées et publiées régulièrement afin d'étudier les évolutions temporelles.

2-Dans le domaine de la recherche, les études sont de nature :

- Epidémiologique : les études sont effectuées dans le cadre du PNSM (études cas témoins et recherche d'autres facteurs étiologiques que l'amiante en particulier chez la femme où la fraction attribuable à une exposition à l'amiante est la plus faible).

- Méthodologique visant à comparer diverses méthodes d'évaluation des expositions.

- Anatomopathologique. Cet autre axe de recherche développe en collaboration avec le PNSM et le Centre National Référent MESOPATH des études d'identification de formes histologiques inhabituelles et/ ou des études de survie et en particulier des longues survies en fonction des aspects histologiques et desexpositions. 
Tableau 2 : nombre de prélèvements reçus par département durant la période 1998-2011

\begin{tabular}{|c|c|c|c|c|c|c|c|c|}
\hline \multirow{3}{*}{$\begin{array}{l}\text { Région (Départements) } \\
\text { Aquitaine }(24,33,40,47,64)\end{array}$} & \multicolumn{8}{|c|}{$\mathrm{Nb}$ de prélèvement reçus par année de prélèvement } \\
\hline & \multicolumn{2}{|c|}{$1998-2002$} & \multicolumn{2}{|c|}{ 2003-2007 } & \multicolumn{2}{|c|}{$2008-2012$} & \multicolumn{2}{|c|}{ Total } \\
\hline & 185 & $19 \%$ & 236 & $17 \%$ & 263 & $15 \%$ & 684 & $17 \%$ \\
\hline Alsace $(67,68)$ & 51 & $5 \%$ & 48 & $4 \%$ & 80 & $4 \%$ & 179 & $4 \%$ \\
\hline Basse-Normandie $(14,50,61)$ & 160 & $16 \%$ & 160 & $12 \%$ & 163 & $9 \%$ & 483 & $12 \%$ \\
\hline Corse $(2 A, 2 B)$ & - & - & 6 & $1 \%$ & 15 & $1 \%$ & 21 & $1 \%$ \\
\hline Franche-Comté (25) & 14 & $2 \%$ & 13 & $1 \%$ & 21 & $1 \%$ & 48 & $1 \%$ \\
\hline Haute-Normandie (76) & - & - & 85 & $6 \%$ & 204 & $12 \%$ & 289 & $7 \%$ \\
\hline Ule-de-France $(93,94)$ & 150 & $15 \%$ & 209 & $15 \%$ & 186 & $11 \%$ & 545 & $13 \%$ \\
\hline Nord-Pas de Calais (59 - région lilloise) & - & - & - & - & 14 & $1 \%$ & 14 & $<1 \%$ \\
\hline Pays-de-la-Loire (44) & 131 & $13 \%$ & 134 & $10 \%$ & 150 & $9 \%$ & 415 & $10 \%$ \\
\hline Picardie (80) & 22 & $2 \%$ & 45 & $3 \%$ & 40 & $2 \%$ & 107 & $3 \%$ \\
\hline Provence-Alpes-Côte d'Azur $(06,13,83)$ & 171 & $17 \%$ & 329 & $24 \%$ & 495 & $28 \%$ & 995 & $24 \%$ \\
\hline Rhône-Alpes (38) & 112 & $11 \%$ & 95 & $7 \%$ & 131 & $7 \%$ & 338 & $8 \%$ \\
\hline Total & 996 & $100 \%$ & 1360 & $100 \%$ & 1762 & $100 \%$ & 4118 & $100 \%$ \\
\hline
\end{tabular}

4-Le Centre Expert National Anatomopathologique "Cancers rares " pour les Mésothéliomes malins pleuraux et les tumeurs péritonéales rares : CNR MESOPATH

En 2009, le CNR MESOPATH a été sélectionné par l'INCa en réponse à la mesure 20 action 20.3 visant à soutenir la qualité en Anatomie et Cytologie pathologique ainsi qu'à la mesure 23 action 23.1 visant àlabelliser les centres de référence pour les cancers rares.

Le CNR MESOPATH est localisé à Caen sous la coordination du Pr F. Galateau-Sallé. Il est organisé par grandes régions, et s'appuie le plus souvent sur les régions possédant des registres de cancers (généraux ou spécialisés). II est constitué pour l'organisation de la deuxième lecture d'un réseau de 15 experts régionaux répartis sur le territoire français(Figure 3 ). Ce réseau d'experts couvre les départements géographiques du PNSM et du registre MESONATmais également les départements dits" hors PNSM »auxquels vient s'ajouter le centre de compétence de Nouméa en Nouvelle Calédonie.

Chaque site régional est sous la responsabilité d'un expert du groupe MESOPATH (Figures 4a et 4b). Cet expert structure son réseau régional grâce à la collaboration des pathologistes du secteur public, semi-privé et privé provenant des 22 départements du PNSM, et de l'ensemble des départements du hors PNSM (France entière et territoires ultramarins). Les suspicions de mésothéliomedes patients provenant de départements non couverts par un site régional sont directement adressées au centre référent national.

Les objectifs principaux du CNR MESOPATH (PNSM et hors PNSM) sont d'organiser la relecture des cas suspectés de mésothéliomeet sont centrés sur la certification anatomopathologique standardisée du diagnostic. Cette relecture est différente de celle des trois autres CNR cancers rares anatomopathologiques labellisés par l'INCa car elle s'appuiesur la procédure de certificationformalisée précédemment décrite (9) mise en place et validée par le PNSM en 1998. Chaque expert régional assure une première relecture des cas signalés, et communique son avis diagnostique au pathologiste initial (envoi d'un compte rendu histologique) afin que le patient puisse 
257 être pris en charge dans les plus brefs délais, puis il adresse le dossier anatomopathologique (blocs

258 de paraffine, ou lames blanches) accompagné de la feuilleannotée de renseignements cliniques au 259 centre réfèrent national MESOPATH qui assure l'organisation de la relecture par trois experts en 260 aveugle des renseignements cliniques et du statutd'exposition à l'amiante. Lorsque les avis de trois experts sont concordants le diagnostic de mésothéliome est définitivement validé ou exclu. Les cas de diagnostics incertains (lorsque l'expertise nepermet pas d'affirmer ou d'exclure définitivement le diagnostic de mésothéliome) sont soumis à une expertise collégiale qui a lieu une fois par mois à Paris avec les experts du CNR MESOPATH (le quorum nécessite la présence de 10 experts). La procédure de relecture implique que tous les cas arrivant à cette étape soient de nouveau examinés au microscope par chaque expert de manière indépendante sans avoir connaissance du contexte clinique ni de celui de l'exposition. A la fin de la séance tous les cas sontdiscutés au microscope multitête. Une décision collégiale est prise ensuite après avoir pris connaissance des éléments connus du dossier clinique mais en dehors de la notion d'exposition à l'amiante. Le diagnostic est obtenu à la majorité des votes.

Résultats

Le tableau 1 correspond à l'activité totale du CNR MESOPATH pour la période 1998-2012incluant les départements du PNSM et ceux du Hors PNSM (résultats enregistrés dans la base de données du CNR MESOPATH pour les prélèvements de la plèvre et du péritoine, la vaginale testiculaire et le péricarde étant exclus de ce tableau.

Tableau 3 ; nombre total de cas revus de 1998 à 2012 en fonction des catégories histologiques les plus fréquentes pour les deux localisations principales : plèvre et péritoine. 


\begin{tabular}{|c|c|c|c|c|}
\hline \multirow{2}{*}{ Histologie 1998-2012 } & \multicolumn{2}{|c|}{ Plèvre } & \multicolumn{2}{|c|}{ Péritoine } \\
\hline & $\mathbf{N}$ & $\%$ & $\mathbf{N}$ & $\%$ \\
\hline Mésothéliome malin & 6250 & $75 \%$ & 381 & $68 \%$ \\
\hline Epithélioïde & 5003 & $80 \%$ & 347 & $91 \%$ \\
\hline Biphasique & 703 & $11 \%$ & 27 & $7 \%$ \\
\hline Sarcomatoïde & 397 & $6 \%$ & 6 & $2 \%$ \\
\hline Desmoplastique & 147 & $2 \%$ & 1 & $<1 \%$ \\
\hline Autre tumeur d'origine mésothéliale & 43 & $1 \%$ & 53 & $10 \%$ \\
\hline Mésothéliome papillaire superficiel bien différencié & 43 & $100 \%$ & 40 & $75 \%$ \\
\hline Mésothéliome multikystique & 0 & $0 \%$ & 13 & $25 \%$ \\
\hline Tumeur adénomatoïde & 0 & $0 \%$ & 0 & $0 \%$ \\
\hline Tumeur fibreuse solitaire & 0 & $0 \%$ & 0 & $0 \%$ \\
\hline Incertain & 710 & $9 \%$ & 29 & $5 \%$ \\
\hline Prolifération mésothéliale de malignité indéterminée & 175 & $25 \%$ & 8 & $27 \%$ \\
\hline Tumeur inclassée & 421 & $59 \%$ & 17 & $59 \%$ \\
\hline Autre & 114 & $16 \%$ & 4 & $14 \%$ \\
\hline Exclus pour autre diagnostic & 946 & $11 \%$ & 83 & $15 \%$ \\
\hline Diagnostic bénin & 282 & $30 \%$ & 16 & $19 \%$ \\
\hline Métastase & 575 & $61 \%$ & 55 & $66 \%$ \\
\hline Autre tumeur primitive & 89 & $9 \%$ & 12 & $15 \%$ \\
\hline Exclus pour matériel insuffisant & 359 & $4 \%$ & 11 & $2 \%$ \\
\hline Total revus & 8308 & $100 \%$ & 557 & $100 \%$ \\
\hline
\end{tabular}

Les types de prélèvements reçus au CNR sont des prélèvements non chirurgicaux (biopsie pleurale ou ponction transpariétale radioguidée) pour $42 \%$ et chirurgicaux (biopsies chirurgicales, pièces opératoires ou prélèvements réalisés sous vidéothoracoscopie)pour $58 \%$ des cas. Les prélèvements cytologiques sont exceptionnels compte tenu des recommandations faites il y a 10 ans de ne pas certifier définitivement un diagnostic de mésothéliome sur une seule cytologie. L'analyse immunohistochimique est systématique et incontournable. Les modalités de cette analyse ont été précédemment décrites (9). Les analyses moléculaires selon les recommandations de I'INCa sont effectuées systématiquement au CNR MESOPATH: recherche de translocation spécifique de sarcomes pour le diagnostic différentiel avec les mésothéliomes sarcomatoïdes ou technique FISH pour la recherche de la délétion de p16 dans les cas de prolifération mesothéliale de malignité indéterminée (HMA).Le matériel jugé insuffisant correspond pour la plupart du temps à des problèmes secondaires à une fixation défectueuse (sous fixation/fixation dans l'AFA/ BOUIN/nouveaux fixateurs) ou à un matériel trop superficiel ou mal inclus, ne permettant pas de juger de l'envahissement des plans profonds. Par souci de précautionpour les patients et leurs ayant droits, pour éviter toute perte de matériel ou de destruction de blocs devant ce cancer rare aux implicationsmédicosociales fortes, et compte tenu de la découverte récente de marqueurs de prédisposition (ex:BAP1), l'archivage des lames histologiques et des blocs de paraffine est réalisé au sein du CNR MESOPATH afin de constituer un archivagede qualité et sécurisé et permettre dans un avenir proche des études moléculaires pertinentes pour une meilleure prise en charge des patients. 
Pour répondre à des objectifs d'efficacité et de rapidité des réponses en toute sécurité, un réseau virtuel national de relecture systématique des lames histologiques a été mis en place à l'aide d'un logiciel d'expertise s'appuyant sur le réseau d'experts MÉSOPATH et le réseau d'experts internationaux du centre d'excellence international par e@pathologieIM@EC. Cette relecture par trois experts du CNR MESOPATH (PNSM et Hors PNSM) est en production depuis juin 2011. Les lames histologiques (HES et 4 à 6 analyses immunohistochimiques) de chaque cas signalé au CNR MESOPATH sont numérisées sur un scanner haut débit (300 lames/24h/). Puis les images sont sauvegardées sur un serveur sécurisé localisé au CHU de Caen. Le logiciel dédié (SGL CCITI) envoie alors un message par mail à trois experts sélectionnés de manière aléatoire. Les experts se connectent grâce à un identifiant et à un mot de passe. Ils s'enregistrent et peuvent ensuite avec le SGL d'expertise CCITI réaliser leur expertise en ligne. Les experts doivent répondre sous $48 \mathrm{~h}$ à la demande d'expertise qui leur est faite. Sinon le cas est systématiquement redistribué à un nouvel expert. A partir du moment où l'expert accepte de prendre en charge l'expertise de ce cas, la réponse doit être retourné via le SGL sous 5 jours.

Résultats

Depuis le 1er juin 2011, date de mise en production du SGL CCITI, 2478 dossiers constitués de 20677 lames (HES et lames d'analyses immunohistochimiques) ont été scannées et envoyées sur le système pour un volume de 5,77 tera octet (TO). Le délai moyen de lecture par les trois experts pour un cas est de 10 jours, le délai minimum observé pour un diagnostic final est de $50 \mathrm{mn}$.

Parmi les 2282 dossiers examinés par trois experts la concordance avec le pathologiste initial est observée dans $74 \%$ des cas, tandis qu'une discordance majeure est notée dans $11 \%$ des dossiers (lésion bénigne versus lésion maligne ou carcinome versus sarcome versus mésothéliome). Les discordances observées par le système de relecture à partir des lames numérisées sont très voisines de celles qui ont été notées lorsque la relecture était effectuée de manière conventionnelle à partir du microscope. L'avantage de ce système est qu'il facilite la communication entre les experts qui peuvent visualiser la lésionet les zones d'intérêt. Ces zones d'intérêt peuvent être conservées, échangées et discutées avec d'autres experts nationaux ou internationaux.

5-Le centre d'excellence international sur le mésothéliome MESOPATH-IM@EC (Panel International des Mésothéliomes)

Le panel international des mésothéliomes a été initié dès 1998lors de la mise en place du PNSM, coordonné par I'INVS. Ses objectifs d'amélioration du diagnostic anatomopathologique sont donc étroitement liés à ceux du PNSM et/ou du CNR MESOPATH.Ce panel réunit un groupe de 22 experts répartis dans le monde entier (Angleterre,Australie, Autriche, Belgique, Canada, Ecosse, France, Japon, Suisse, USA) (Figure 5).Les objectifs principaux du panel international sont d'examiner et de discuter les dossiers difficiles, de répertorier et d'analyser les tumeurs inclassées identifiées parmi les diagnostics incertains, depuis 1998, dans la base de données du PNSM et du CNR et de définir des critères morphologiques immunohistochimiques et moléculaires ( $\mathrm{AMH}$ project) permettant 
des guidelines et recommandations internationales pour la prise en charge des mésothéliomes malins. Les experts du panel international sont équipés du même SGL d'expertise pour l'analyse des lames numérisées(MESOPATH IM@EC). Le Panel International des Mésothéliomes est un groupe affilié à I'US \&Canadian Academy of Pathology (USCAP) et se réunit tous les ans à l'occasion du meeting de I'USCAP, voire une deuxième fois dans l'année à l'occasion d'une réunion de I'AIP (Academy International of Pathology), du College of American Pathologists (CAP), de l'International Mesothelioma Interest Group (IMIG) ou d'une réunion d'une autre Société Européenne ou internationale.Les travaux réalisés au cours de ces 15 années ont permis la publication d'une monographie (10), d'articles de "recommandations de bonne pratique pour la prise en charge des mésothéliomes" en langue anglaise (11-14) et de plusieurs articles sur les modalités diagnostiques des lésions bénignes versus malignes (15) ou sur de nouvelles formes histologiques de mésothéliome (16-18).

\section{6-La base clinicobiologique MESOBANK pour la recherche épidémiologique et translationnelle sur} le mésothéliome

En septembre 2011, créé à l'initiative et avec le soutien de l'Institut National du Cancer suite à l'appel d'offre lancé en juillet 2011, le projet MESOBANK a proposé de donner aux efforts déjà réalisés par le PNSM/le registre MESONAT/le CNR MESOPATH, une nouvelle dimension en constituant une véritable biobanque virtuelle nationale exhaustive sur le mésothéliome (Figure 5). Les enjeux de ce dernier appel d'offre pour le mésothéliome étant d'optimiser la base clinico-biologique, épidémiologique, thérapeutique et de suivi déjà initiée afin de structurer une collection d'échantillons de haute qualité susceptible d'être utilisée à des fins de recherche fondamentale ou translationnelle. La base de données clinico-biologique de MESOBANK est articulée avec celle du Centre Référent National sur les mésothéliomes malins pleuraux et les tumeurs péritonéales rares MESOPATH soutenu par I'INCa. La base est étroitement interconnectée au Programme National de Surveillance des Mésothéliomes [PNSM], au Registre multicentrique à vocation Nationale des mésotheliomes MESONAT et au Centre d'Excellence Internationale MESOPATH-IM@EC (groupe d'experts internationaux spécialistes du mésothéliome provenant des États Unis, Canada, Europe, Australie, Japon). La MESOBANK comprend une partie tissulaire pilotée par F. Galateau Sallé et une partie lignées cellulaires pilotée par MC Jaurand (UMR U674 J.Zucman-Rossi). Cette MESOBANK regroupe tous les prélèvements dont le diagnostic est certifié selon la procédure standardisée collégiale, de haute qualité, à reconnaissance internationale, et à un potentiel élevé pour développer une activité de recherche translationelle.

Les objectifs de MESOBANK sont (1) de structurer et d'optimiser les bases des tumorothèques des 10 centres de ressources biologiques [CRB] soutenus par I'INCa en une seule et unique base multicentrique à vocation nationale ; (2) de développer des projets collaboratifs ambitieux exploitant le matériel collecté et visant à la découverte et la validation de nouveaux marqueurs de détection précocesen collaboration avec les centres nationaux référents cliniques MESOCLIN ( Centre Expert Clinique National mésotheliomes malins pleuraux)et RENAPE ( Centre Expert Clinique National cancers rares du péritoine)et avec les groupes coopérateurs cliniques français; (3) de permettre la 
mise en place de projets de grande ampleur en particulier dans le domaine de la génomique (séquençage d'exomes complets).

L'intérêt de constituer la MESOBANK et de promouvoir son exploitation est d'obtenir une base riche sur le plan clinique et épidémiologique aisément requêtable sur le plan national, facilitant la mise en place de projets collaboratifs. Dix projets, financés par ailleurs, utilisant différentes technologies analysant les altérations moléculaires et les voies de signalisation sur les bases moléculaires de l'initiation et de la progression tumorale du mésothéliome sont actuellement en cours de développement. Cette base de données va faciliter et augmenter l'impact des collaborations internationales initiées par MESOPATH en vue de la prochaine classification histologique internationale de I'OMS [WHO] sur le mésothéliome ainsi que de la prochaine stadification TNM de I'UICC en collaboration en collaboration avec I'IASLC (recherche de marqueurs pronostiques)(19). Enfin, cette ressource va soutenir de nouveaux programmes de recherche fondamentale en génomique CGH ARRAY projet libre du Cancéropole NO et projet de séquençage international : $\mathrm{Pr}$ Bruce Robinson et en pharmacogénomique, les PHRC nationaux BIO-MAPS et MESOTHEL sur les états précancéreux de la plèvre pour aboutir, à terme, à une meilleure détection et une meilleure prise en charge diagnostique et pronostique de ces patients.

Résultats

Localement le pathologiste régional cryopréserve dans la tumorothèque ou le CRB régional les échantillons miroir des blocs de paraffine des cas signalés lorsque cela est possible. Les liquides d'épanchements en fonction des sites sont également cryopréservés. L'atteinte de l'objectif implique la structuration d'un CRB national virtuel"MESOBANK". Cette structuration est en cours de développement.7021 blocs de paraffine sont stockés dans la MESOBANK. 684 patients pour lesquels le diagnostic de mésothéliome a été certifié selon la procédure standardisée ont des prélèvements cryopréservés, enregistrés dans le CRB MESOBANK constitués de 4711 échantillons, de 3996 échantillons d'épanchements et de 59 cultures cellulaires.

Les études moléculaires récentes effectuées à partir des cellules et des tissus ont identifié des biomarqueurs sériques (mésothéline, ostéopontine, calrétinine MUC1, A.Scherpereel, des marqueurs de classification pronostique(p16, IMP3, EMA, M.Karanian-Philippe/ F.Galateau-Sallé) $(20,21)$ et des biomarqueurs de détection précoce comme la recherche de la délétion du gène de CDKN2A ( $p 16$ INK4A) par FISH sur les hyperplasies mésothéliales atypiques : suspicion d'états précancéreux de la plèvre et du péritoine (21). D'autres biomarqueurs potentiels sont également en cours d'étude, y compris la détection d'anomalies chromosomiques (CGH ARRAY, MC Jaurand sur les cellules, $F$ Galateau Sallé sur les tissus, et Martin Figeac IFR 114 de Lille(22-24), des modifications de voies de signalisation (Cmet Levallet et al.(25), des altérations de l'expression de p53 et de NF2 (Panel International- F.Galateau-Sallé /P. Hainaut/MC Jaurand(26) et des altérations de l'environnement tumoral et du contexte immunologique (M.Paciencia/ A.Scherpereel). Toutes ces investigations contribuent à mieux comprendre les bases moléculaires du mésothéliome de façon à mieux assurer sa détection précoce et sa prise en charge thérapeutique.

Lien entre le réseau des pathologistes et les CNR cliniques MESOCLIN et RENAPE et le réseau RING

Les liens avec le CNR MESOCLIN sont extrêmement forts par la participation des pathologistes aux réunions de concertation pluridisciplinaire pour le mésothéliome pleural institué dans chaque région 
réunissant, physiquement ou par « Webconférence " selon les distances, les pathologistes référents de chaque centre expert pour la prise en charge des patients. Le CNR MESOPATH participe à la " RCP virtuelle » nationale de recours par l'intermédiaire d'un serveur dédié [logiciel de RCP virtuelle créé dans ce but] en lien avec le site de l'Intergroupe Francophone de Cancérologie Thoracique (IFCT), permettant à tout clinicien de déposer les données anonymisées cliniques et d'imagerie (TDM, TEPTDM, thoracoscopie...) et aux pathologistes de répondre ou de poser des questionsaux cliniciens en cas de problèmes diagnostiques. Par ailleurs, le centre expert national MESOCLIN et MESOPATH constitueront avec l'aide de I'INCa et de I'IFCT un site de téléchargement spécifique pour le mésothéliome de documents PDF (conférence de consensus, articles, protocoles...).

La mise en place d'une étroite collaboration avec nos partenaires du réseau expert MESOCLIN et de I'IFCT permet d'incrémenter la banque nationale de données cliniques et d'imagerie pour promouvoir la recherche clinique, épidémiologique et translationnelle dans le mésothéliome malin pleural, en lien avec la biobanque virtuelle nationale du consortium mésothéliome "Mesobank" affichée sur le site de l'INCa.

La collaboration étroite instaurée avec le réseau RENAPE et le réseau des pathologistes RENAPATH permet d'homogénéiser la classification histologique des mésothéliomes entre la plèvre et le péritoine, et de mieux répertorier les cas de tumeurs péritonéales rares. Deux réunions annuelles ont été organisées conjointement avec les cliniciens et une réunion de consensus avec les pathologistes à Paris.

La réunion avec le réseau RING des pathologistes spécialistes de la sphère génitale a été extrêmement fructueuse, et a permis de clarifier les diagnostics de certains types histologiques, et d'améliorer les discordances majeures entre experts (figures6 et 7).

\title{
Place des pathologistes
}

Les pathologistes ont joué depuis 1998 et jouent un rôle essentiel au sein du réseau et sont un maillon incontournable du dispositif de surveillance de cette maladie à déclaration obligatoire (voir ci-dessus), dans la reconnaissance de cette pathologie au titre des maladies professionnelles pour les patients qui ont été exposés, mais également dans la participation active à la constitution de la base de données clinicobiologiques.En effet, les $\mathrm{CRHs}$ détaillés et les fiches de renseignements cliniques annotées permettent d'enrichir continuellement cette base de données unique par la qualité et le nombre des données.

\section{Comment contacter le réseau}

Le centre MESOPATH est localisé au CHU de Caen au sein du Laboratoire d'Anatomie Pathologique Pour les pathologistes les blocs d'inclusion en paraffine ou les lames blanches si le bloc est épuisé doivent être adressés au:

\author{
Pr. Françoise Galateau-Sallé \\ Laboratoire d'Anatomie Pathologique \\ CHU Caen Cote de Nacre \\ 14033 Caen cedex. \\ Tel : 0231064407 \\ Fax : 0231065063 \\ Mail : galateausalle-f@chu-caen.fr
}


Pour la réception et le secrétariat :

Adélaide Obin

obin-a@chu-caen.fr

Pour l'enregistrement des dossiers :

Pour le suivi des dossiers:

492

\section{Pour le PNSM}

\section{Pour la plateforme numérique}

Thomas Rousvoal

Tel:02 31064812

Fax:02 31065063

rousvoal-t@chu-caen.fr

Pour les conventions, les projets, les questions réglementaires, la qualitéet la MESOBANK.

Gaetane Blaizot

Tel : 0231064405 -

Fax : 0231065063

blaizot-g@chu-caen.fr

Pour les analyses statistiques et les données du registre MESONAT.

Nolwenn Le Stang

Tel : 0231064405

Fax : 0231065063

lestang-n@chu-caen.fr

\section{Coordination Générale}

Anabelle Gilg Soit Ilg

Département Santé Travail INSTITUT DE VEILLE SANITAIRE

12 rue du Val d'Osne

94415 Saint-Maurice Cedex France

Tél. : 33 (0)1 41796819

Fax : 33 (0)1 41796788

http://www.invs.sante.fr

a.gilg@invs.sante.fr 


\section{Centre médico-social}

\section{Centre exposition}

Céline. Gramond Sabyne Audignon ISPED-LSTE-ESSAT

146 Rue Leo Saignat 33076 Bordeaux Cedex Tél. : 33 (0)5 57574780 Fax : 33 (0)5 57574733 Celine.gramond@isped.u-bordeaux2.fr Sabyne.audignon@isped.u-bordeaux2.fr

\section{Pourla Déclaration obligatoire de maladie [DO]}

Dr Laurence Chérié-Challine

Département Santé Travail INSTITUT DE VEILLE SANITAIRE

12 rue du Val d'Osne 94415 Saint-Maurice Cedex France Tél. : 33 (0)1 55125415 Fax : 33 (0)1 41796788 http://www.invs.sante.fr I.cheriechalline@invs.sante.fr

Les principales tumeurs prises en charge par le réseau concernent les tumeurs primitives incidentes de la plèvre pour le PNSM, les mésothéliomes malins des séreuses quel que soit le site (plèvre, péritoine, vaginale testiculaire et péricarde)et les tumeurs péritonéales rares pour certification diagnostique. 
579 Ce réseau mésothéliome structuré autour d'équipes multidisciplinaires depuis 15 ans a fait toute la

580

581

582

583

584

585

586

587

588

589

590

591

592

593

594

595

596

597

598

599

600

601

602

603

604

605

606

607

608

609

610

611

612

613

614

615

616

617

618

619 preuve de son intérêt et de son efficacité pour produire des données et des indicateurs de surveillance épidémiologique au niveau national. Le réseau des pathologistes a montré tout l'intérêt qu'il y avait à mettre en place cette relecture et cette procédure de certification standardisée anatomopathologique pour assurer la qualité des données épidémiologiques, la qualité de la reconnaissance médicosociale et la fiabilité du diagnostic clinique pour le bénéfice des patients. Ce réseau multidisciplinaire novateur,internationalementreconnu a permis au fur et à mesure des années de mettre en place une base de données clinico-biologiques a potentiel élevé pour développer des activités de recherche translationnelle et épidémiologique.

Remerciements

Nous remercions vivement Marie Christine Petit, Céline Fleury, Solange Leblanc, Jacqueline Hoyez, Adelaide Obin, Annick Tardiff, Magalie Leval, pour leur assistance technique constante, les techniciennes des différents laboratoires, les enquêteurs, les chargés d'études et tous ceux qui participent de près ou de loin à ce réseau, trop nombreux pour être cités et dont la collaboration est essentielle au bon fonctionnement et aux résultats du réseau.

\section{Références}

1. Peto J, Hodgson JT, Matthews Fe,Jones JR. Continuing increase in mesothelioma mortality in Britain. Lancet. 1995 4;345:535-9

2. Banaei $A$, Auvert $B$, Goldberg $M$ et al.Future trends in mortality of French men from mesothelioma.Occup Environ Med. 2000 Jul;57(7):488-94)

3. Le Stang N, Belot A, Gilg Soit Ilg A, Rolland P, Astoul P, Bara S, Brochard P,Danzon A, Delafosse P, Grosclaude $P$, Guizard AV, Imbernon E, Lapôtre-Ledoux B, Ligier K, Molinié F, Pairon JC, Sauleau EA, Trétarre B, Velten M, Bossard N,Goldberg M, Launoy G, Galateau-Sallé F.Evolution of pleural cancers and malignant pleural mesothelioma incidence in France between 1980 and 2005.Int J Cancer. 2010 Jan 1;126(1):232-8.

4. Pairon JC, Laurent F, Rinaldo $M$, Clin B, Andujar P, Ameille J, Brochard P, Chammings S, Ferretti G, Galateau-Sallé F, Gislard A, Letourneux M, Luc A, Schorlé E, Paris C. Pleural plaques and the risk of pleural mesothelioma. J Natl Cancer Inst. 2013 Feb 20;105(4):293-301.

5. Rolland P, Gramond C, Briand A, Astoul P, Chamming's S, Ducamp S, Frenay C, Galateau-Sallé G, Gilg Soit Ilg A, Imbernon E, Le Stang N, Pairon JC, Goldberg G, Brochard P. Occupations and industries in France at high risk for pleural mesothelioma: a population-based case-control study (1998-2002). Am J Ind Med 2010, 2010 Dec;53(12):1207-19.

6. Lacourt A, Rolland P, Gramond C, Astoul P, Chamming's S, Ducamp S, Frenay C, Galateau-Sallé F, Gilg Soit Ilg A, Imbernon E, Le Stang N, Pairon JC, Goldberg M, Iwatsubo Y, Salmi LR, Brochard P. Attributable risk in men in two French case-control studies on mesothelioma and asbestos. Eur J Epidemiol. 2010 Sep 7.

7. Gilg Soit Ilg A, Goldberg M, Rolland P, Chamming's S, Ducamps S et al. Programme national de surveillance du mésothéliome - Principaux résultats 1998-2006. Saint-Maurice (Fra) : Institut de veille sanitaire, juillet 2009, 24 p. Disponible sur : www.invs.sante.fr

8. Chamming's S, Clin B, Brochard P, Astoul P, Ducamp S, Galateau-Salle F, Ilg AG, Goldberg M, Gramond C, Imbernon E, Rolland P, Pairon JC. Compensation of pleural mesothelioma in France: 
Data from the French National Mesothelioma Surveillance Programme. Am J Ind Med. 2012 Aug 21. doi: 10.1002/ajim.22106.

9. Goldberg $M$, Imbernon $E$, Rolland $P$, Gilg Soit Ilg $A$, Savès $M$, de Quillacq $A$, Frenay $C$, Chamming's S, Arveux P, Boutin C, Launoy G, Pairon JC, Astoul P, Galateau-Sallé F, Brochard P. The French National Mesothelioma Surveillance Program. Occup Environ Med. 2006 Jun;63(6):390-5. Epub 2006 Feb 9

10. Monography: Pathology of malignant mesothelioma; An update of the International Mesothelioma Panel. Editeur F. Galateau-Sallé Springer Verlag.2006

11. Husain AN, Colby T, Ordonez N, Krausz T, Attanoos R, Beasley MB, Borczuk AC, Butnor K, Cagle PT, Chirieac LR, Churg A, Dacic S, Fraire A, Galateau-Salle F, Gibbs A, Gown A, Hammar S, Litzky L, Marchevsky AM, Nicholson A, Roggli V, Travis WD, Wick M. Guidelines for Pathologic Diagnosis of Malignant Mesothelioma: 2012 Update of the Consensus Statement from the International Mesothelioma Interest Group. Arch Pathol Lab Med. 2012 Aug 28.

12. Husain AN, Colby TV, Ordóñez NG, Krausz T, Borczuk A, Cagle PT, Chirieac LR, Churg A, GalateauSalle F, Gibbs AR, Gown AM, Hammar SP, Litzky LA, Roggli VL, Travis WD, Wick MR.Guidelines for pathologic diagnosis of malignant mesothelioma: a consensus statement from the International Mesothelioma Interest Group. Arch Pathol Lab Med. 2009 Aug;133(8):1317-31.

13. Bellocq JP, Arnould L, Chenard MP, Ettore F, Galateau-Sallé F, Michenet P, Michiels JF, Molina T, Penault-Llorca F. Recommendations and quality control in immunohistochemistry. Ann Pathol. 2009 Nov;29 Spec No 1:S96-7. Epub 2009 Oct 21.

14. Scherpereel A, Astoul P, Baas P, Berghmans T, Clayson H, de Vuyst P, Dienemann $H$, GalateauSalle F, Hennequin C, Hillerdal G, Le Péchoux C, Mutti L, Pairon JC, Stahel R, van Houtte P, van Meerbeeck J, Waller D, Weder W.Guidelines of the European Respiratory Society and the European Society of Thoracic Surgeons for the management of malignant pleural mesothelioma. Eur Respir J. 2010 Mar;35(3):479-95. Epub 2009 Aug 28.

15. Churg A, Galateau-Salle F.The separation of benign and malignant mesothelial proliferations. Arch Pathol Lab Med. 2012 Oct;136(10):1217-26.

16. Galateau-Sallé F, Attanoos R, Gibbs AR, Burke. L, Astoul.P, Rolland.P, Gilg soit Ilg A, Pairon JC, Brochard $P$, Begueret $H$, Vignaud JM, Kerr K, Launoy $G$, Imbernon E, Goldberg $M$. Lymphohistiocytoid variant of malignant mesothelioma of the pleura: A series of 22 cases. Am J Surg Pathol, $200731 ; 5: 711-6$.

17. Galateau-Sallé F, Copin MC, Delajartre AY, Vignaud JM, Astoul P, Pairon JC, Le Pimpec-Barthes F, Brochard P. [Which criteria for the anatomopathologic diagnosis of malignant pleural mesothelioma?]. Rev Mal Respir. 2006 Sep;23(4 Pt :11S37-44.

18. Galateau-Sallé F, Vignaud JM, Burke L, Gibbs A, Brambilla E, Attanoos R, Goldberg M, Launoy G; Mesopath group. Well-differentiated papillary mesothelioma of the pleura: a series of 24 cases. Am J Surg Pathol. 2004 Apr;28(4):534-40.

19. Rice D, Rusch V, Pass H, Asamura H, Nakano T, Edwards J, Giroux DJ, Hasegawa S, Kernstine KH, Waller D, Rami-Porta R; International Association for the Study of Lung Cancer International Staging Committee and the International Mesothelioma Interest Group. Recommendations for uniform definitions of surgical techniques for malignant pleural mesothelioma: a consensus report of the international association for the study of lung cancer international staging committee and the international mesothelioma interest group. J Thorac Oncol. 2011 Aug;6(8):1304-12. 
20. de Reyniès $A$, JauranD MC, Renier A, Couchy G, Hysi, Elarouci N, Galateau-Sallé F, Copin $M C$, Hofman P, Cazes A, Andujar P, Imbeaud S, Petel F, Pairon JC, Le Pimpec-Barthes F, Zucman-Rossi J, Didier J. Molecular classification of malignant pleural mesothelioma: Identification ofa poor prognosis subgroup linked to the epithelial-to-mesenchymal transition. Clinical Cancer Research accepté.

21. Dacic S, Kothmaier H, Land S, Shuai Y, Halbwedl I, Morbini P, Murer B, Comin C, Galateau-Salle F, Demirag $F$, Zeren $H$, Attanoos $R$, Gibbs A, Cagle $P$, Popper $H$. Prognostic significance of p16/cdkn2a loss in pleural malignant mesotheliomas. Virchows Arch. 2008 Dec;453(6):627-35.

22. Kothmaier H, Quehenberger F, Halbwedl I, Morbini P, Demirag F, Zeren H, Comin CE, Murer B, Cagle PT, Attanoos R, Gibbs AR, Galateau-Salle F, Popper HH. EGFR and PDGFR differentially promote growth in malignant epithelioid mesothelioma of short and long term survivors. Thorax. 2008 Apr;63(4):345-51.

23. Jean D, Daubriac J, Le Pimpec-Barthes F, Galateau-Salle F, Jaurand MC. Molecular changes in mesothelioma with an impact on prognosis and treatment. Arch Pathol Lab Med. 2012 Mar;136(3):277-93.

24. Weinbreck N, Vignaud JM, Begueret H, Burke L, Benhattar J, Guillou L, Capron F, Galateau-Salle F. SYT -SSX fusion is absent in sarcomatoid mesothelioma allowing its distinction from synovial sarcoma. Mod Pathol 2007;20:617-21

25. Levallet G, Vaisse-Lesteven M, Le Stang N, Ilg AG, Brochard P, Astoul P, Pairon JC, Bergot E, Zalcman $G$, Galateau-Sallé F. Plasma cell membrane localization of c-MET predicts longer survival in patients with malignant mesothelioma: a series of 157 cases from the MESOPATH Group. J Thorac Oncol. 2012 Mar;7(3):599-606.

26. Andujar P, Pairon JC, Renier A, Descatha A, Hysi I, Abd-Alsamad I, Billon-Galland MA, Blons H, Clin B, Danel C, Debrosse D, Galateau-Sallé F, Housset B, Laurent-Puig P, Le Pimpec Barthes F, Letourneux M, Monnet I, Régnard JF, Validire $P$, Zucman-Rossi J, Jaurand MC, Jean D. Differential mutation profiles and similar intronic TP53 polymorphisms in asbestos-related lung cancer and pleural mesothelioma. Mutagenesis. 2013 Feb 22 
Figure 2 Carte des départements couverts par le registre MESONAT

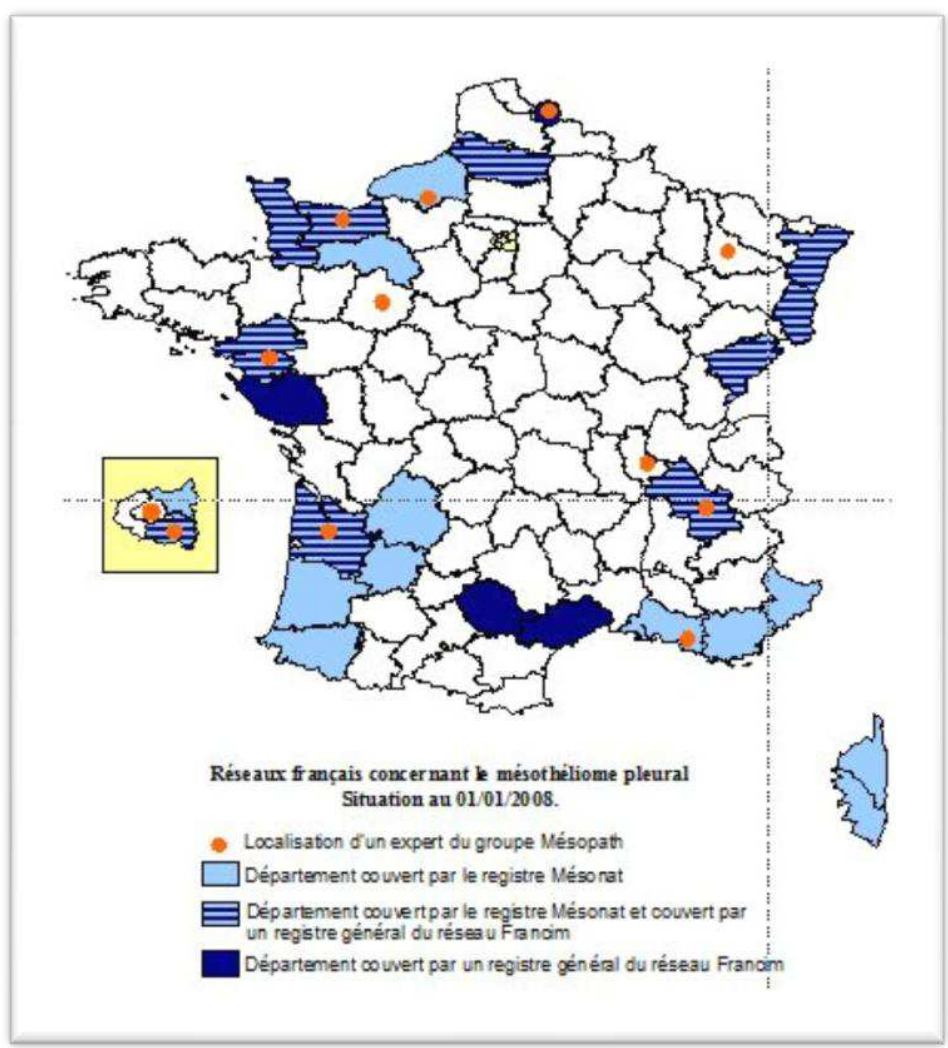


Figure 3 : Carte des départements couverts par le CNR MESOPATH

720

721



Figure 4 : Liste des experts MESOPATH( 4A et 4B) 


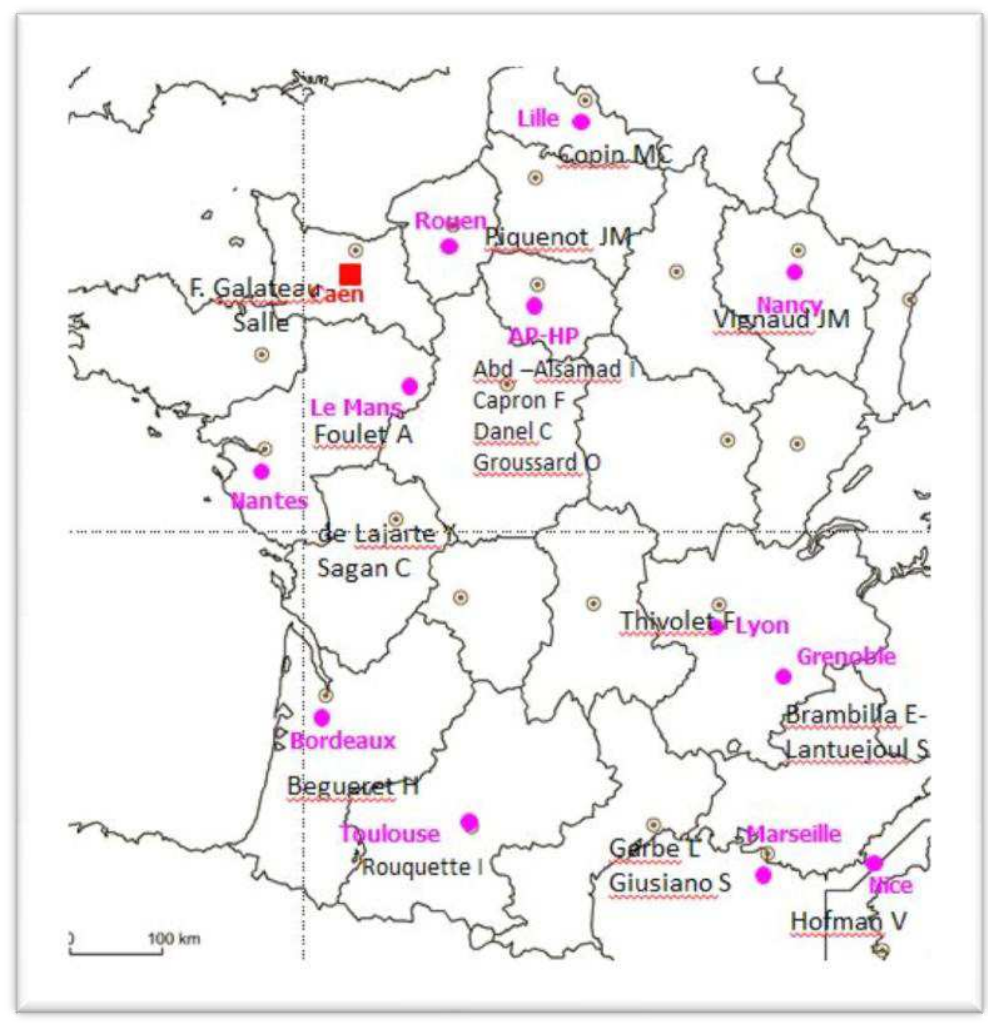

724

\begin{tabular}{lll}
\hline Experts & Centre régional & $\begin{array}{l}\text { Année } \\
\text { d'entrée }\end{array}$ \\
\hline I abdalsamad & IDF & 1995 \\
\hline H. Begueret & Aquitaine & 1995 \\
\hline E Brambilla & Grenoble & 1995 \\
\hline F Capron & IDF & 1981 \\
\hline MC Copin & Nord Pas de Calais & 2000 \\
\hline C Danel & IDF & 2011 \\
\hline AY de Lajartre & Loire Atlantique & 1972 \\
\hline E Foulet- Roge & Sarthe & 2000 \\
\hline F Galateau Salle & CNR national & 1982 \\
\hline L Garbe & PACA & 1990 \\
\hline O Groussard & IDF & 1995 \\
\hline S Giusiano & PACA & 2013 \\
\hline V Hofman & PACA & 2011 \\
\hline S Lantuejoul & Rhône Alpes & 2011 \\
\hline JM Piquenot & Haute Normandie & 1995 \\
\hline I Rouquette & Garonne & 2011 \\
\hline S Sagan & Loire Atlantique & 2008 \\
\hline F Thivolet-Bejui & Rhône Alpes & 1995 \\
\hline JM Vignaud & Lorraine & 1995 \\
\hline
\end{tabular}


728

729

730

731 


\section{Internatıonal IVlesothelıoma txcellence Center}

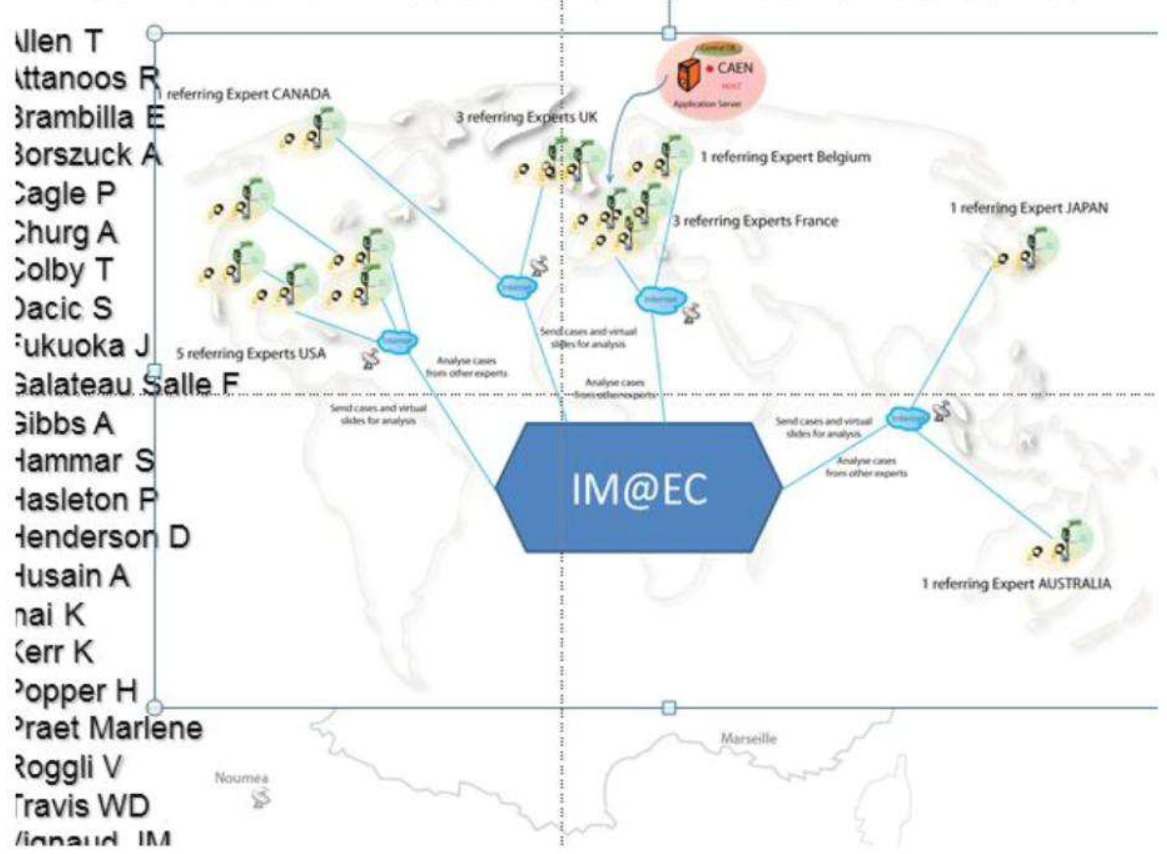

Figure 6 : MESOBANK

\section{MESOBANK}

Base clinicobiologique multicentrique à visée nationale pour la recherche épidémiologique et translationnelle Taille de la base $=70 \mathrm{M}$

Outils: nombre de lignes attendues (14000 dossiers patients, 1200 pts/an

MESOBANK -suprabase
TVN du consortium
mesothéliome

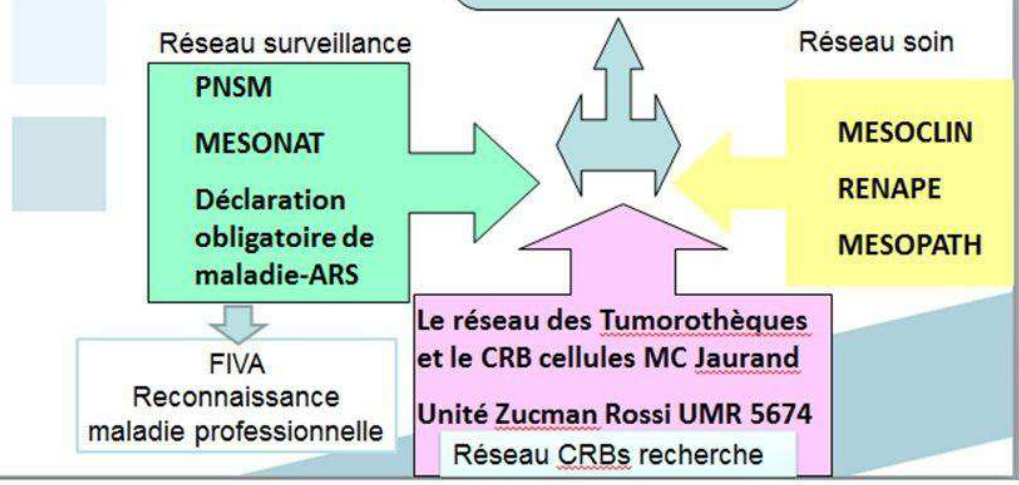




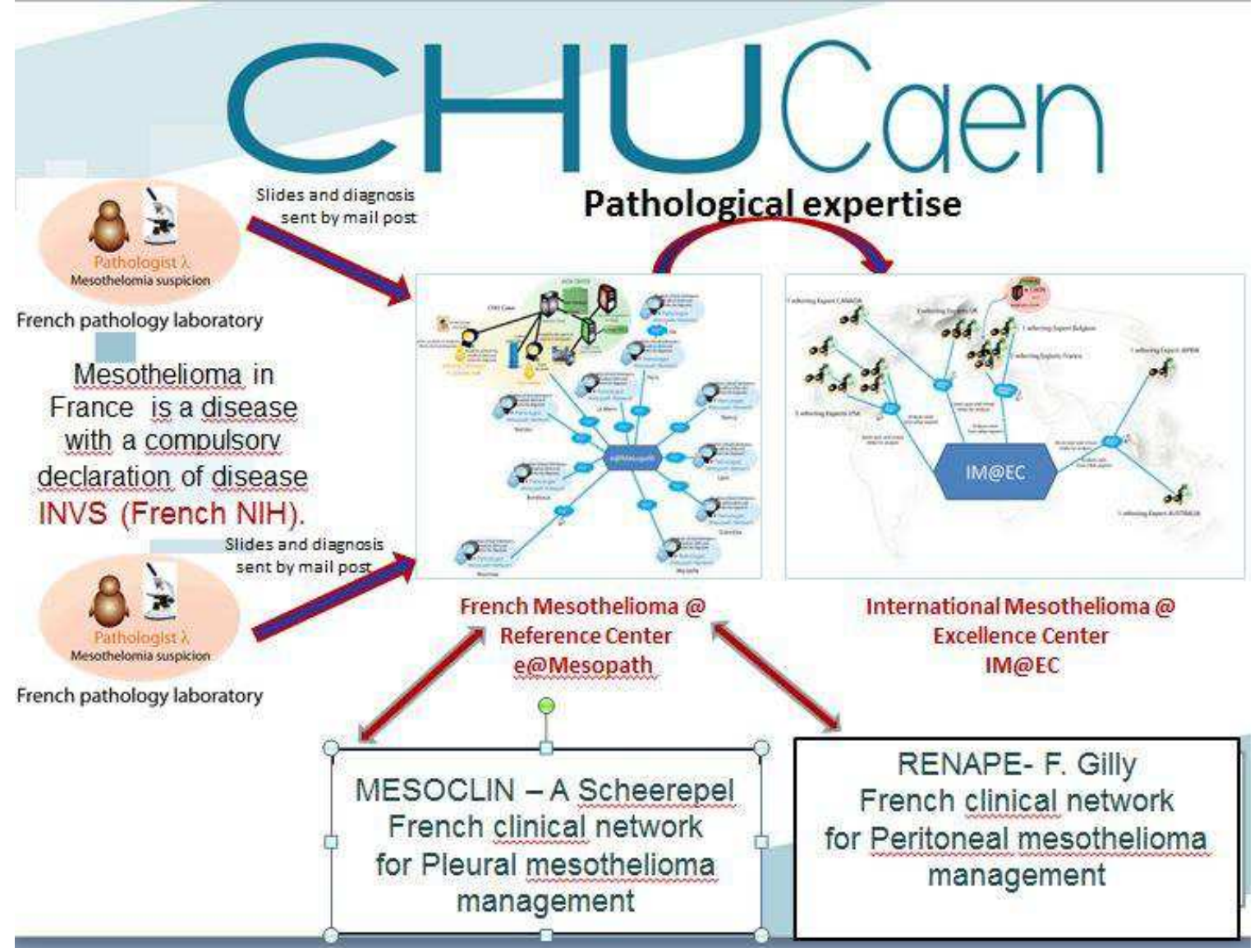

